\title{
Radar Waveform Strategy Based on Game Theory
}

\author{
Honglin $W A N G^{1}$, Wei $L I^{1,2}$, He $W A N G^{3}$, Jianye $X U^{1}$, Junlong ZHAO ${ }^{1}$ \\ ${ }^{1}$ Information and Navigation College, Air Force Engineering University, Fenghao Road 1st Xi'an 710077, China \\ ${ }^{2}$ Collaborative Innovation Center of Information Sensing and Understanding, Fenghao Road 1st Xi' an 710077, China \\ ${ }^{3}$ Dept. of Basic Sciences, Air Force Engineering University, Changle Road 1st Xi'an 710051, China
}

wanghonglin821@outlook.com, liweichangsha@163.com,18066540235@163.com,xjy1236@sina.com, 1170755712@qq.com

Submitted March 24, 2019 / Accepted September 21, 2019

\begin{abstract}
In this paper, we proposed two waveform design methods based on game theory to address the problem of radar detection performance degradation in electronic warfare. Since radar and jammer are completely hostile, their interaction is modeled as two-person zero-sum game. Signal-to-Interference-plus-Noise Ratio (SINR) criterion is used in formulating the utility functions. The existence of Nash equilibrium in games is verified by mathematical derivation. Different game waveform strategies are designed for different information levels of radar and jammer. Iterative water-filling method and two-step waterfilling method are designed to achieve Cournot equilibrium and Stackelberg equilibrium, respectively. Simulation results reveal that game strategies can bring higher radar detection performance than No game signal, especially when jammer power is lower than radar power. Radar detection probability based on game theory can be increased by up to $10 \%$ without changing the power. This demonstrates game strategies have great potentials for radar waveform design in electronic warfare.
\end{abstract}

\section{Keywords}

Game theory, waveform strategy, Cournot game, Stackelberg game, Nash equilibrium, Signal-toInterference-plus-Noise Ratio (SINR)

\section{Introduction}

Optimizing radar transmit waveform is a key way to improve radar detection performance [1-9]. With the rapid development of electronic warfare technology, the confrontation between radar and jammer has become increasingly fierce. Cognitive radar can adaptively optimize transmit waveform based on prior knowledge [10]. But at the same time, enemy jammer intercepts radar waveform and releases jamming pertinently, resulting in a decline in radar performance. [11]. Therefore, optimizing the waveform to improve radar detection performance has become a significant research topic.
Game theory has long been the subject of many classic studies in economics, politics and biology. Recent research has validated that game theory can provide a useful account of improving radar system performance. In 2011, Gogineni and Nehorai first applied game theory to the radar field. They proposed a polarimetric waveform design scheme for distributed MIMO radar based on game theory [12]. In later work conducted by Panoui and Deligiannis, the power allocation schemes of Multistatic MIMO radar network based on different game models were investigated. Generalized Nash equilibrium, Stackelberg Nash equilibrium and Bayesian Nash equilibrium power strategies were solved to achieve high signal-to-noise ratio (SNR) gain and save power resources [13-15]. Considering the joint design of amplitudes and frequency-hopping codes for frequencyhopping waveforms, Han proposed a game theory framework to improve MIMO radar performance [16]. The distributed power control problem of radar network is formulated as cooperative game and non-cooperative game in [17], [18], which effectively improves the low probability of intercept (LPI) performance of radar network. Overall, these studies support the notion that game theory can improve radar system performance by optimizing signal or power. Such approaches, however, have failed to address the effects of jamming on radar. The degradation of radar performance caused by enemy jamming is a problem that must be considered in modern electronic warfare.

It is only since the work of Song et al. that the game theory in radar and jammer has gained momentum [19]. They studied the two-person zero-sum game model between MIMO radar and jammer and designed Stackelberg equilibrium solution based on mutual information (MI) criterion. Gao Hao studied the Bayesian game strategy of MIMO radar and jammer from the perspective of incomplete information [20]. But the effects of clutter are not taken into account. Actually, power allocation strategies are usually affected by clutter in electronic warfare. In our former work, Stackelberg game between MIMO radar and jammer in the presence of clutter was studied [21]. The optimization scheme based on MI criterion was explored and analyzed. To date, some published articles have begun to focus on the game phenomenon between radar and jam- 
mer, but such studies remain narrow in focus dealing only with MIMO radar. Unfortunately, the operational mechanism of SISO/SIMO radar is different from MIMO radar. Besides, the game scheme based on MI criterion cannot be well applied to non-Gaussian clutter environment. With the aim of improving SISO/SIMO radar detection performance in electronic warfare, this paper proposed waveform game strategies between radar and jammer in clutter based on SINR criterion.

Here, we propose radar waveform design methods from the perspective of game theory. Since jammer and radar are completely hostile, their interaction is modeled as two-person zero-sum game. Nash equilibriums in games are verified by mathematical derivation. To analyze the feasibility of the game waveform design method in different scenarios, two game models are considered as following: 1) Cournot Game Model (radar and jammer have equal status), and 2) Stackelberg Game Model (jammer has a late-mover advantage). To the best of our knowledge, this is the first time to propose SISO/SIMO radar waveform design strategies based on game theory in clutter. This method provides a stable solution for improving radar detection performance in electronic warfare. Both the theoretical analysis and numerical calculations demonstrate that radar performance can be improved through game strategies.

\section{Signal Model}

Figure 1 shows a block diagram of radar signal model used for SINR-based waveform design. Here, $s(t)$ is the complex-valued transmit waveform with finite-energy, duration $T$, and Fourier transform $S(t) . r(t)$ is the complexvalued receive filter impulse response. $h(t)$ is the extended known target impulse response of finite duration $T_{\mathrm{h}}$ and Fourier transform $H(f) . n(t)$ is a complex-valued, zeromean channel noise process with power spectral density (PSD) $S_{\mathrm{nn}}(f)$, which is non-zero over the entire waveform bandwidth. $c(t)$ is a complex-valued, random process representing a clutter component, e.g., ground clutter, and characterized by the PSD $S_{\mathrm{cc}}(f)$. $S_{\mathrm{nn}}(f)$ and $S_{\mathrm{cc}}(f)$ are positive in the signal bandwidth $W$ (i.e., the frequency band $[-W / 2, W / 2])$. Let jamming signal $j(t)$ be the finite-energy waveform with finite duration $T_{\mathrm{h}}$ and Fourier transform $J(f)$. As seen in Fig. 1, the signal $y(t)$ at the output of the receive filter is

$$
y(t)=r(t) *[s(t) * h(t)+s(t) * c(t)+n(t)+j(t)] .
$$

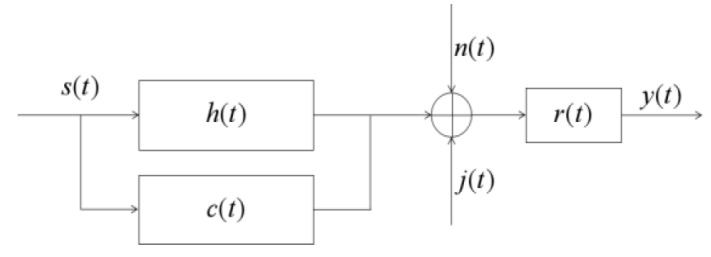

Fig. 1. Radar signal model under electronic warfare conditions. $y_{\mathrm{s}}(t)$ and $y_{\mathrm{j}}(t)$ are the signal and jamming components of the output $y(t)$, respectively, which are defined by

$$
y_{\mathrm{s}}(t)=r(t) * s(t) * h(t)
$$

and

$$
y_{\mathrm{j}}(t)=r(t) *[s(t) * c(t)+n(t)+j(t)] .
$$

The output SINR at time $t_{0}$ is

$$
\begin{aligned}
(\operatorname{SINR})_{t_{0}}= & \frac{\left|y_{\mathrm{s}}\left(t_{0}\right)\right|^{2}}{E\left(\left|y_{i}\left(t_{0}\right)\right|^{2}\right)}= \\
& \frac{\left|\int_{-\infty}^{+\infty} R(f) H(f) S(f) \mathrm{e}^{\mathrm{j} 2 \pi f t_{0}} \mathrm{~d} f\right|^{2}}{\int_{-\infty}^{+\infty}|R(f)|^{2}\left(S_{\mathrm{cc}}(f)|S(f)|^{2}+J(f)+S_{\mathrm{nn}}(f)\right) \mathrm{d} f}
\end{aligned}
$$

where $h(t)$ is a complex wide-sense stationary process. We define

$$
\sigma_{\mathrm{h}}^{2}(f)=E\left(\left|H(f)-\mu_{\mathrm{h}}(f)\right|^{2}\right)
$$

as the variance of $H(f)$, called the energy spectral variance (ESV). Here $\mu_{\mathrm{h}}(f)$ is the mean of $H(f)$, assuming $\mu_{\mathrm{h}}(f)=0$ The ESV describes the average energy of a finite-duration, zero-mean process in the same sense that a PSD describes the average power of an infinite-duration, wide-sense stationary process [4]. Put (5) into (4) and apply Schwarz's inequality yields the bound:

$$
\begin{aligned}
&(\mathrm{SINR})_{t_{0}} \leq\left\{\int_{-\infty}^{+\infty}|R(f)|^{2}\left(S_{\mathrm{cc}}(f)|S(f)|^{2}+J(f)+S_{\mathrm{nn}}(f)\right) \mathrm{d} f\right. \\
&\left.\int_{-\infty}^{+\infty} \frac{\sigma_{\mathrm{h}}^{2}(f)|S(f)|^{2}}{S_{\mathrm{cc}}(f)|S(f)|^{2}+J(f)+S_{\mathrm{nn}}(f)} \mathrm{d} f\right\} . \\
&\left\{\int_{-\infty}^{+\infty}|R(f)|^{2}\left(S_{\mathrm{cc}}(f)|S(f)|^{2}+J(f)+S_{\mathrm{nn}}(f)\right) \mathrm{d} f\right\}^{-1} .
\end{aligned}
$$

The SINR achieves its maximum of

$$
(\mathrm{SINR})_{t_{0}}=\int_{-\infty}^{+\infty} \frac{\sigma_{\mathrm{h}}^{2}(f)|S(f)|^{2}}{S_{\mathrm{cc}}(f)|S(f)|^{2}+J(f)+S_{\mathrm{nn}}(f)} \mathrm{d} f
$$

if and only if the matched filter is of the form

$$
R(f)=\frac{\left[k H(f) S(f) \mathrm{e}^{\mathrm{j} 2 \pi t_{0}}\right]^{*}}{S_{\mathrm{cc}}(f)|S(f)|^{2}+J(f)+S_{\mathrm{nn}}(f)}
$$

where $k$ is an arbitrary constant. To visually represent the distributed power of the radar waveform on each sub-band, SINR may be converted from integral to discrete:

$$
\mathrm{SINR} \simeq \Delta f \sum_{k=1}^{K} \frac{\sigma_{\mathrm{h}}^{2}\left(f_{k}\right)\left|S\left(f_{k}\right)\right|^{2}}{S_{\mathrm{cc}}\left(f_{k}\right)\left|S\left(f_{k}\right)\right|^{2}+J\left(f_{k}\right)+S_{\mathrm{nn}}\left(f_{k}\right)}
$$

where $K$ is the frequency sample number, and $\Delta f$ is the frequency sampling interval.

\section{Waveform Strategy}

This section assumes that both radar and jammer are 
cognitive. Some prior knowledge can be used to adaptively optimize waveforms. Signal-to-Interference-plus-Noise Ratio (SINR) criterion is used in formulating the utility functions.

\subsection{No Game}

Firstly, radar and jammer unilateral optimal schemes are given. Assume that the power of radar and jammer is $P_{\mathrm{S}}$ and $P_{\mathrm{J}}$, respectively; the waveform power constraint may be written as

$$
\Delta f \sum_{k=1}^{K}\left|S\left(f_{k}\right)\right|^{2} \leq P_{\mathrm{S}}, \quad \Delta f \sum_{k=1}^{K} J\left(f_{k}\right) \leq P_{\mathrm{J}} .
$$

\subsubsection{Radar Unilateral Strategy}

In the radar unilateral model, when radar makes a decision, it does not know the jammer's decision. For reasons of conservation and rationality, radar assumes that jammer releases Gaussian white noise jamming in signal band, which does not change the target characteristics. For this case, to maximize SINR, radar can choose the following strategy to optimize the transmit waveform:

$$
\left\{\begin{array}{l}
\max _{\left|S\left(f_{k}\right)\right|^{2}} \Delta f \sum_{k=1}^{K} \frac{\sigma_{\mathrm{h}}^{2}\left(f_{k}\right)\left|S\left(f_{k}\right)\right|^{2}}{S_{\mathrm{cc}}\left(f_{k}\right)\left|S\left(f_{k}\right)\right|^{2}+J\left(f_{k}\right)+S_{\mathrm{nn}}\left(f_{k}\right)} \\
\text { s.t. } \Delta f \sum_{k=1}^{K}\left|S\left(f_{k}\right)\right|^{2} \leq P_{\mathrm{s}}, J\left(f_{k}\right)=\frac{P_{\mathrm{J}}}{K \Delta f}, k=1,2, \cdots K .
\end{array}\right.
$$

Notice that the objective function is a convex function of $\left|S\left(f_{k}\right)\right|^{2}$, and the power constraint is linear. Therefore, an optimal scheme can be obtained by the water-filling method [1]. Considering the objective function and constraints, the waveform optimization problem is transformed into a convex programming problem, which is solved by Lagrange multiplier method:

$$
\begin{aligned}
L\left(\left|S\left(f_{k}\right)\right|^{2}, \lambda\right)= & \Delta f \sum_{k=1}^{K} \frac{\sigma_{\mathrm{h}}^{2}\left(f_{k}\right)\left|S\left(f_{k}\right)\right|^{2}}{S_{\mathrm{cc}}\left(f_{k}\right)\left|S\left(f_{k}\right)\right|^{2}+J\left(f_{k}\right)+S_{\mathrm{nn}}\left(f_{k}\right)}+ \\
& \lambda\left(P_{\mathrm{s}}-\Delta f \sum_{k=1}^{K}\left|S\left(f_{k}\right)\right|^{2}\right) .
\end{aligned}
$$

Take $L\left(\left|S\left(f_{k}\right)\right|^{2}, \lambda\right)$ 's derivative and make it equal to zero to maximize SINR. We can get the radar optimized signal:

$$
\begin{aligned}
& \left|S\left(f_{k}\right)\right|^{2}= \\
& \left(\sqrt{\frac{\sigma_{\mathrm{h}}^{2}\left(f_{k}\right)\left(J\left(f_{k}\right)+S_{\mathrm{nn}}\left(f_{k}\right)\right)}{S_{\mathrm{cc}}^{2}\left(f_{k}\right) \lambda}}-\frac{J\left(f_{k}\right)+S_{\mathrm{nn}}\left(f_{k}\right)}{S_{\mathrm{cc}}\left(f_{k}\right)}\right)^{+}
\end{aligned}
$$

where $(x)^{+} \triangleq \max \{0, x\} \quad, \quad \lambda>0 \quad$ is determined by $\Delta f \sum_{k=1}^{K}\left|S\left(f_{k}\right)\right|^{2}=P_{\mathrm{s}}$.

\subsubsection{Jammer Unilateral Strategy}

Suppose the jammer knows radar's income function (maximizing SINR). In order to degrade radar performance, jammer needs to optimize the jamming signal based on the detected radar waveform parameters. Assuming that radar waveform spectrum is stable, the jamming waveform design strategy can be:

$$
\left\{\begin{array}{l}
\min _{J\left(f_{k}\right)} \Delta f \sum_{k=1}^{K} \frac{\sigma_{\mathrm{h}}^{2}\left(f_{k}\right)\left|S\left(f_{k}\right)\right|^{2}}{S_{\mathrm{cc}}\left(f_{k}\right)\left|S\left(f_{k}\right)\right|^{2}+J\left(f_{k}\right)+S_{\mathrm{nn}}\left(f_{k}\right)} \\
\text { s.t. } \Delta f \sum_{k=1}^{K} J\left(f_{k}\right) \leq P_{\mathrm{J}},\left|S\left(f_{k}\right)\right|^{2}=\frac{P_{\mathrm{s}}}{K \Delta f}, k=1,2, \ldots, K .
\end{array}\right.
$$

The kernel in (14) is monotonically decreasing and concave, and power constraint of $P_{\mathrm{J}}$ is linear, so the optimization solution is unique. The jamming waveform can be obtained by the water-filling method:

$$
\begin{aligned}
& L\left(J\left(f_{k}\right), \gamma\right)= \\
& \Delta f \sum_{k=1}^{K} \frac{\sigma_{\mathrm{h}}^{2}\left(f_{k}\right)\left|S\left(f_{k}\right)\right|^{2}}{S_{\mathrm{cc}}\left(f_{k}\right)\left|S\left(f_{k}\right)\right|^{2}+J\left(f_{k}\right)+S_{\mathrm{nn}}\left(f_{k}\right)}+\gamma\left(P_{\mathrm{J}}-\Delta f \sum_{k=1}^{K} J\left(f_{k}\right)\right) .
\end{aligned}
$$

Solve the optimized jamming waveform

$$
\begin{aligned}
& J\left(f_{k}\right)= \\
& \left(\sqrt{\frac{\sigma_{\mathrm{h}}^{2}\left(f_{k}\right)\left|S\left(f_{k}\right)\right|^{2}}{\gamma}}-S_{\mathrm{cc}}\left(f_{k}\right)\left|S\left(f_{k}\right)\right|^{2}-S_{\mathrm{nn}}\left(f_{k}\right)\right)^{+} .
\end{aligned}
$$

Without considering the game, the final strategy of radar and jammer is as follows:

$$
\left\{\begin{array}{l}
\left|S\left(f_{k}\right)\right|^{2}=\left(\sqrt{\frac{\sigma_{\mathrm{h}}^{2}\left(f_{k}\right)\left(J\left(f_{k}\right)+S_{\mathrm{nn}}\left(f_{k}\right)\right)}{S_{\mathrm{cc}}^{2}\left(f_{k}\right) \lambda}}-\frac{J\left(f_{k}\right)+S_{\mathrm{nn}}\left(f_{k}\right)}{S_{\mathrm{cc}}\left(f_{k}\right)}\right)^{+} \\
\text {s.t. } \Delta f \sum_{k=1}^{K}\left|S\left(f_{k}\right)\right|^{2} \leq P_{\mathrm{s}}, J\left(f_{k}\right)=\frac{P_{\mathrm{J}}}{K \Delta f}, k=1,2, \cdots K \\
J\left(f_{k}\right)=\left(\sqrt{\frac{\sigma_{\mathrm{h}}^{2}\left(f_{k}\right)\left|S\left(f_{k}\right)\right|^{2}}{\gamma}}-S_{\mathrm{cc}}\left(f_{k}\right)\left|S\left(f_{k}\right)\right|^{2}-S_{\mathrm{nn}}\left(f_{k}\right)\right)^{+} \\
\text {s.t. } \Delta f \sum_{k=1}^{K} J\left(f_{k}\right) \leq P_{\mathrm{J}},\left|S\left(f_{k}\right)\right|^{2}=\frac{P_{\mathrm{s}}}{K \Delta f}, k=1,2, \ldots, K
\end{array}\right.
$$

\subsection{Cournot Game}

In the electronic warfare, the relationship between radar and jammer is detection and anti-detection. Both action strategies can be explained by game model.

The Cournot model was proposed by the French economist Antoine Augustin Cournot in 1838. The model illustrates how the production decisions of firms that compete with each other and do not coordinate with each other produce a result that lies between the competitive equilibrium and the monopoly equilibrium [22]. Ref. [23] analyzed the game equilibria always exist if two agents have symmetric information. If a game is competitive and has a unique pure-strategy Nash equilibrium, all the players 
prefer to stay at Nash equilibrium under the assumptions of conservativeness and rationality. In the Cournot game, radar and jammer have symmetric information and equal status. Therefore, it is significant to analyze Nash equilibrium strategy between radar and jammer.

In order to solve the optimization problem in Cournot game, we first need to find and prove the game equilibrium solution between radar and jammer. The optimization scheme has to satisfy the following four characteristics to ensure equilibrium [19]:

(1) If $\left|S\left(f_{k}\right)\right|^{2}=0$, then $J\left(f_{k}\right)=0$; if $J\left(f_{k}\right) \neq 0$, then $\left|S\left(f_{k}\right)\right|^{2} \neq 0$.

(2) For any two sub-bands $f_{m}$ and $f_{n}$, and $J\left(f_{n}\right)>0$, $J\left(f_{m}\right)>0$.

(A) If $\frac{\sigma_{\mathrm{h}}^{2}\left(f_{m}\right)}{S_{\mathrm{cc}}^{2}\left(f_{m}\right)}>\frac{\sigma_{\mathrm{h}}^{2}\left(f_{n}\right)}{S_{\mathrm{cc}}^{2}\left(f_{n}\right)}$, then

$$
J\left(f_{m}\right)+S_{\mathrm{nn}}\left(f_{m}\right)>J\left(f_{n}\right)+S_{\mathrm{nn}}\left(f_{n}\right) ;
$$

(B) If $\frac{\sigma_{\mathrm{h}}^{2}\left(f_{m}\right)}{S_{\mathrm{cc}}^{2}\left(f_{m}\right)}=\frac{\sigma_{\mathrm{h}}^{2}\left(f_{n}\right)}{S_{\mathrm{cc}}^{2}\left(f_{n}\right)}$, then

$$
J\left(f_{m}\right)+S_{\mathrm{nn}}\left(f_{m}\right)=J\left(f_{n}\right)+S_{\mathrm{nn}}\left(f_{n}\right) ;
$$

(C) If $\frac{\sigma_{\mathrm{h}}^{2}\left(f_{m}\right)}{S_{\mathrm{cc}}^{2}\left(f_{m}\right)}<\frac{\sigma_{\mathrm{h}}^{2}\left(f_{n}\right)}{S_{\mathrm{cc}}^{2}\left(f_{n}\right)}$, then

$$
J\left(f_{m}\right)+S_{\mathrm{nn}}\left(f_{m}\right)<J\left(f_{n}\right)+S_{\mathrm{nn}}\left(f_{n}\right) .
$$

(3) For any two sub-bands $f_{m}$ and $f_{n}$, if $J\left(f_{m}\right)>0$ and $J\left(f_{n}\right)=0$, then $J\left(f_{m}\right)+S_{\mathrm{nn}}\left(f_{m}\right)<S_{\mathrm{nn}}\left(f_{n}\right)$.

(4) For any two sub-bands $f_{m}$ and $f_{n}$, and $\frac{\sigma_{\mathrm{h}}^{2}\left(f_{m}\right)}{S_{\mathrm{cc}}^{2}\left(f_{m}\right)} \geq \frac{\sigma_{\mathrm{h}}^{2}\left(f_{n}\right)}{S_{\mathrm{cc}}^{2}\left(f_{n}\right)}, S_{\mathrm{nn}}\left(f_{m}\right)<S_{\mathrm{nn}}\left(f_{n}\right)$, if $J\left(f_{n}\right)>0$, then $J\left(f_{m}\right)>0$.

Proof: When the game reaches the equilibrium point, for reasonable reasons, if there is no signal power in a subband $f_{k}$, the jammer will not allocate any jamming power in the sub-band, otherwise it will waste a limited amount of jamming power. In order to optimize the interference performance, the jammer will distribute jamming power in the sub-band which has radar signal power. Therefore, the characteristic (1) is verified.

When $J\left(f_{k}\right)>0, \quad\left|S\left(f_{k}\right)\right|^{2}>0$ can be obtained from characteristic (1). So

$$
\begin{aligned}
& \frac{\sigma_{\mathrm{h}}^{2}\left(f_{k}\right)\left|S\left(f_{k}\right)\right|^{2}}{S_{\mathrm{cc}}\left(f_{k}\right)\left|S\left(f_{k}\right)\right|^{2}+J\left(f_{k}\right)+S_{\mathrm{nn}}\left(f_{k}\right)}= \\
& \frac{\sigma_{\mathrm{h}}^{2}\left(f_{k}\right)}{S_{\mathrm{cc}}\left(f_{k}\right)}-\frac{\sqrt{\lambda \sigma_{\mathrm{h}}^{2}\left(f_{k}\right)\left(J\left(f_{k}\right)+S_{\mathrm{nn}}\left(f_{k}\right)\right)}}{S_{\mathrm{cc}}\left(f_{k}\right)} .
\end{aligned}
$$

Let $x_{k}=J\left(f_{k}\right)+S_{\mathrm{nn}}\left(f_{k}\right)$, then the contribution of $x_{k}$ to the objective function (19) on sub-band $f_{k}$ is

$$
g_{k}\left(x_{k}\right)=\frac{\sigma_{\mathrm{h}}^{2}\left(f_{k}\right)}{S_{\mathrm{cc}}\left(f_{k}\right)}-\frac{\sqrt{\lambda \sigma_{\mathrm{h}}^{2}\left(f_{k}\right) x_{k}}}{S_{\mathrm{cc}}\left(f_{k}\right)} .
$$

When $x_{k}>0$ and $\lambda>0$, we have

$$
\frac{\partial g_{k}\left(x_{k}\right)}{\partial x_{k}}=-\frac{\sqrt{\lambda \sigma_{\mathrm{h}}^{2}\left(f_{k}\right)}}{2 S_{\mathrm{cc}}\left(f_{k}\right) \sqrt{x_{k}}}<0
$$

and

$$
\frac{\partial g_{k}^{2}\left(x_{k}\right)}{\partial x_{k}^{2}}>0
$$

Function $g_{k}\left(x_{k}\right)$ is a concave function with decreasing monotonicity, so

$$
\int_{x_{n}-\Delta}^{x_{n}} \frac{\partial g_{n}(x)}{\partial x} \mathrm{~d} x-\int_{x_{m}}^{x_{m}+\Delta} \frac{\partial g_{m}(x)}{\partial x} \mathrm{~d} x>0
$$

for any two sub-bands $f_{m}$ and $f_{n}$, there is $\frac{\sigma_{\mathrm{h}}^{2}\left(f_{m}\right)}{S_{\mathrm{cc}}^{2}\left(f_{m}\right)}>\frac{\sigma_{\mathrm{h}}^{2}\left(f_{n}\right)}{S_{\mathrm{cc}}^{2}\left(f_{n}\right)}$. Without loss of generality, suppose the optimal solution is $0<x_{m} \leq x_{n}$, defining a nonnegative number $\Delta$, satisfying $0<\Delta<\frac{x_{n}-x_{m}}{2}$, then

$$
\begin{aligned}
& \int_{x_{n}-\Delta}^{x_{n}} \frac{\partial g_{n}(x)}{\partial x} \mathrm{~d} x-\int_{x_{m}}^{x_{m}+\Delta} \frac{\partial g_{m}(x)}{\partial x} \mathrm{~d} x \\
& =\frac{\sqrt{\lambda \sigma_{\mathrm{h}}^{2}\left(f_{n}\right)}}{S_{\mathrm{cc}}\left(f_{n}\right)}\left(\sqrt{x_{n}-\Delta}-\sqrt{x_{n}}\right)-\frac{\sqrt{\lambda \sigma_{\mathrm{h}}^{2}\left(f_{m}\right)}}{S_{\mathrm{cc}}\left(f_{m}\right)}\left(\sqrt{x_{m}}-\sqrt{x_{m}+\Delta}\right) \\
& \leq \frac{\sqrt{\lambda \sigma_{\mathrm{h}}^{2}\left(f_{n}\right)}}{S_{\mathrm{cc}}\left(f_{n}\right)}\left(\sqrt{x_{n}-\Delta}-\sqrt{x_{n}}\right)-\frac{\sqrt{\lambda \sigma_{\mathrm{h}}^{2}\left(f_{m}\right)}}{S_{\mathrm{cc}}\left(f_{m}\right)}\left(\sqrt{x_{n}-\Delta}-\sqrt{x_{n}}\right) \\
& =\sqrt{\lambda}\left(\sqrt{x_{n}-\Delta}-\sqrt{x_{n}}\right)\left(\sqrt{\frac{\sigma_{\mathrm{h}}^{2}\left(f_{m}\right)}{S_{\mathrm{cc}}^{2}\left(f_{m}\right)}}-\sqrt{\frac{\sigma_{\mathrm{h}}^{2}\left(f_{n}\right)}{S_{\mathrm{cc}}^{2}\left(f_{n}\right)}}\right) \leq 0 .
\end{aligned}
$$

Obviously, $x_{m} \leq x_{n}$ cannot be the optimal solutions, which contradicts the assumptions. Therefore, the characteristic A) is proved. The same reason can be proved that characteristics B), C) are established.

Characteristics (3) and (4) can also be proved by the counter-evidence method. Therefore, the Cournot Nash equilibrium exists, and we can achieve it by iterative waterfilling method. For a given jammer's strategy, we obtain the radar's best response strategy via maximizing the radar's utility. Substituting this radar's strategy into the jammer's utility function, we obtain jammer's best response to this radar's strategy via maximizing the jammer's utility. Repeat this process until both radar and jammer strategies remain unchanged. This stable result is Nash equilibrium in game theory. At this equilibrium point, radar and jammer have no incentive to deviate from this point. The algorithm can be summarized as shown in Tab. 1 .

\subsection{Stackelberg Game}

In Sec. 3.2, the status of radar and jammer are equal. Considering the status between radar and jammer during the game may be asymmetrical. In that case, the Cournot model is difficult to apply. Game model established by German economist Heinrich von Stackelberg reflects this asymmetrical competition [22]. As two participants in game 


\begin{tabular}{|c|c|}
\hline \multicolumn{2}{|r|}{ Iterative water-filling algorithm } \\
\hline 1 & $\begin{array}{l}\text { Initialize two players' actions in Section 3.1: } \\
\qquad\left|S\left(f_{k}\right)\right|=\left|S\left(f_{k}\right)\right|_{0}, J\left(f_{k}\right)=J\left(f_{k}\right)_{0}\end{array}$ \\
\hline 2 & $\begin{array}{c}\text { Maximize the radar's utility: } \\
\max _{\operatorname{SINR}}\left(\left|S\left(f_{k}\right)\right|^{*}, \lambda\right)\end{array}$ \\
\hline 3 & $\begin{array}{l}\text { Update the radar's strategy: } \\
\qquad\left|S\left(f_{k}\right)\right|=\left|S\left(f_{k}\right)\right|^{*}\end{array}$ \\
\hline 4 & $\begin{array}{l}\text { Maximize the jammer's utility: } \\
\min _{\operatorname{SINR}}\left(J\left(f_{k}\right)^{*}, \gamma\right)\end{array}$ \\
\hline 5 & $\begin{array}{l}\text { Update the jammer's strategy: } \\
\qquad J\left(f_{k}\right)=J\left(f_{k}\right)^{*}\end{array}$ \\
\hline 6 & $\begin{array}{l}\text { Repeat Step } 2-5 \text { till }\left|S\left(f_{k}\right)\right|^{*} \text { and } J\left(f_{k}\right)^{*} \\
\text { remain unchanged, and the Cournot } \\
\text { equilibrium strategy is }\left\{\left|S\left(f_{k}\right)\right|^{*}, J\left(f_{k}\right)^{*}\right\} .\end{array}$ \\
\hline
\end{tabular}

Tab. 1. Iterative water-filling algorithm.

theory, the leader moves firstly, and the follower makes choice after observing the leader's choice. The leader knows that its strategy will be intercepted by its opponent. With conservativeness and rationality assumptions, the leader adopts the strategy which can alleviate the worst case, and the game yields a Stackelberg equilibrium.

Assume radar is the leader of Stackelberg game. As a follower, the jammer moves later and designs the optimal jamming scheme. To reduce performance losses, radar may adopt the max-min two-step optimization scheme for waveform optimization [21].

$$
\left\{\begin{array}{l}
\max _{\left|S\left(f_{k}\right)\right|^{2}} \min _{J\left(f_{k}\right)} \Delta f \sum_{k=1}^{K} \frac{\sigma_{\mathrm{h}}^{2}\left(f_{k}\right)\left|S\left(f_{k}\right)\right|^{2}}{S_{\mathrm{cc}}\left(f_{k}\right)\left|S\left(f_{k}\right)\right|^{2}+J\left(f_{k}\right)+S_{\mathrm{nn}}\left(f_{k}\right)} \\
\text { s.t. } \Delta f \sum_{k=1}^{K}\left|S\left(f_{k}\right)\right|^{2} \leq P_{\mathrm{s}}, \Delta f \sum_{k=1}^{K} J\left(f_{k}\right) \leq P_{\mathrm{J}}, k=1,2, \cdots K .
\end{array}\right.
$$

The jamming signal in Sec. 3.1 can be applied to the first step of water-filling method, and the radar optimization strategy can be simplified to

$$
\left\{\begin{array}{l}
\max _{\left|S\left(f_{k}\right)\right|^{2}} \Delta f \sum_{k=1}^{K} \frac{\sigma_{\mathrm{h}}^{2}\left(f_{k}\right)\left|S\left(f_{k}\right)\right|^{2}}{S_{\mathrm{cc}}\left(f_{k}\right)\left|S\left(f_{k}\right)\right|^{2}+J\left(f_{k}\right)+S_{\mathrm{nn}}\left(f_{k}\right)} \\
\text { s.t. } J\left(f_{k}\right)=\left(\sqrt{\frac{\sigma_{\mathrm{h}}^{2}\left(f_{k}\right)\left|S\left(f_{k}\right)\right|^{2}}{\gamma}}-S_{\mathrm{cc}}\left(f_{k}\right)\left|S\left(f_{k}\right)\right|^{2}-S_{\mathrm{nn}}\left(f_{k}\right)\right)^{+} \\
\text {s.t. } \Delta f \sum_{k=1}^{K}\left|S\left(f_{k}\right)\right|^{2} \leq P_{\mathrm{s}}, \Delta f \sum_{k=1}^{K} J\left(f_{k}\right)=P_{\mathrm{J}}, k=1,2, \cdots K .
\end{array}\right.
$$

According to the objective function and constraints, the Lagrange equation can be constructed. Optimal solution can be solved by the second step water-filling method.

$$
\begin{aligned}
& L\left(\left|S\left(f_{k}\right)\right|^{2}, \lambda_{2}, \lambda_{3}\right)=\Delta f \sum_{k=1}^{K} \frac{\sigma_{\mathrm{h}}^{2}\left(f_{k}\right)\left|S\left(f_{k}\right)\right|^{2}}{S_{\mathrm{cc}}\left(f_{k}\right)\left|S\left(f_{k}\right)\right|^{2}+J\left(f_{k}\right)+S_{\mathrm{nn}}\left(f_{k}\right)} \\
& +\lambda_{2}\left(J\left(f_{k}\right)-\sqrt{\frac{\sigma_{\mathrm{h}}^{2}\left(f_{k}\right)\left|S\left(f_{k}\right)\right|^{2}}{\gamma}}+S_{\mathrm{cc}}\left(f_{k}\right)\left|S\left(f_{k}\right)\right|^{2}+S_{\mathrm{nn}}\left(f_{k}\right)\right) \\
& +\lambda_{3}\left(P_{\mathrm{s}}-\Delta f \sum_{k=1}^{K}\left|S\left(f_{k}\right)\right|^{2}\right) .
\end{aligned}
$$

The game strategy is as follows:

$$
\left\{\begin{array}{l}
\left|S\left(f_{k}\right)\right|^{2}=\left(\frac{\left(\gamma-\lambda_{2}\right)^{2} \sigma_{\mathrm{h}}^{2}\left(f_{k}\right)}{4 \gamma\left(\lambda_{3}-\lambda_{2} S_{\mathrm{cc}}\left(f_{k}\right)\right)^{2}}\right)^{+} \\
J\left(f_{k}\right)=\left(\sqrt{\frac{\sigma_{\mathrm{h}}^{2}\left(f_{k}\right)\left|S\left(f_{k}\right)\right|^{2}}{\gamma}}-S_{\mathrm{cc}}\left(f_{k}\right)\left|S\left(f_{k}\right)\right|^{2}-S_{\mathrm{nn}}\left(f_{k}\right)\right)^{+} \\
\text {s.t. } \Delta f \sum_{k=1}^{K}\left|S\left(f_{k}\right)\right|^{2} \leq P_{\mathrm{s}}, \Delta f \sum_{k=1}^{K} J\left(f_{k}\right) \leq P_{\mathrm{J}}, k=1,2, \cdots K .
\end{array}\right.
$$

\section{Numerical Results}

In the simulation, we take a certain type of guidance radar as an example. Its signal center frequency is $35 \mathrm{GHz}$, bandwidth is $100 \mathrm{MHz}$ and transmit power is $20 \mathrm{dBw}$. In order to clearly show the power distribution of radar and jammer at each frequency band and simplify the calculation, we divide the entire frequency band into five subbands, each sub-band $\Delta f=20 \mathrm{MHz}$. According to prior knowledge, the PSD of the extended known target is defined $\left\{\sigma_{\mathrm{h}}^{2}\left(f_{k}\right)\right\}=\{1,3,5,7,4\}, \quad S_{\mathrm{nn}}\left(f_{k}\right)=1$, $\left\{S_{\mathrm{cc}}\left(f_{k}\right)\right\}=\{1.2,2,1.5,1,0.8\}$.

\subsection{Jammer Power Fixed}

First, we investigate Nash equilibrium between radar and jammer. Assume that the jammer can accurately estimate the radar signal frequency band and the jamming power is $20 \mathrm{dBw}$. Figure 2 illustrates the game equilibrium exists and is achieved at point E. Figure 3 shows the dynamic iterative process of Nash equilibrium. Point $A$ is the initial position. The SINR versus iteration numbers is shown in Fig. 4. After 4 iterations, all players cannot improve their own benefits by changing their strategy individually. The Cournot game eventually reached equilibrium with the SINR of $9.782 \mathrm{~dB}$. This shows that the equilibrium strategies are the best choice for conservative and rational radar and jammer. At the equilibrium point, both radar and jammer have no incentive to deviate this point. 


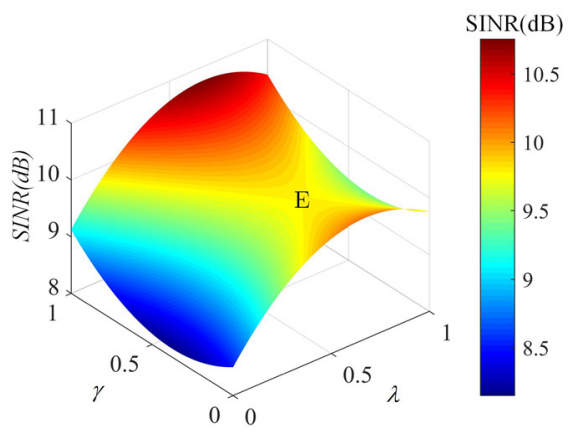

Fig. 2. The SINR versus different strategies of the radar and the jammer.

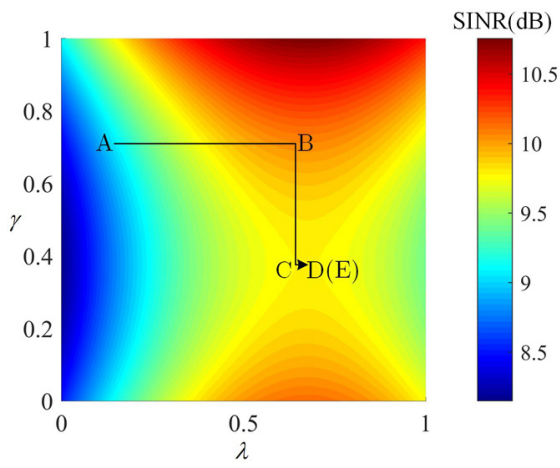

Fig. 3. The dynamic process to achieve the Nash equilibrium in the Cournot game.

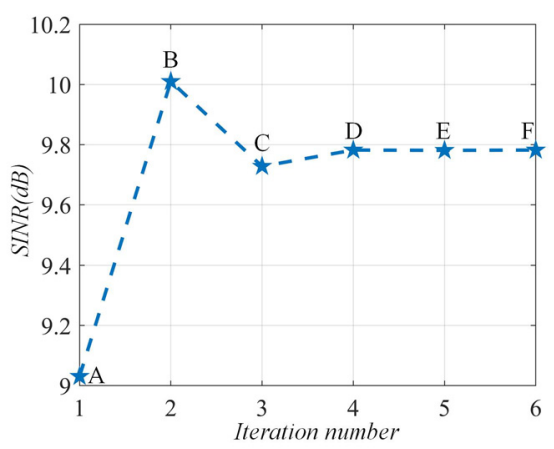

Fig. 4. The SINR versus iteration numbers in Cournot game.

Next, power distribution in frequency domain is carried out for different waveforms. Based on the NeymanPearson theory, assuming the radar false alarm probability is $10^{-4}$, we can derive the radar detection probability by SINR. As can be seen from Fig. 5 and Fig. 6, different signal waveforms result in different power allocation strategies. When the radar does not consider the game with the jammer, the radar designs the optimal waveform according to prior knowledge. It can be seen that compared with chirp signal, the radar signal optimized based on SINR criterion allocates more energy in the frequency band of high target impulse response, low clutter and noise.

In Cournot game, two players take into account each other's strategies when formulating the waveform strategy. First, radar optimizes signal to maximize SINR. Then, to minimize SINR, the jammer designs jamming waveform according to the radar signal. After several iterations, the
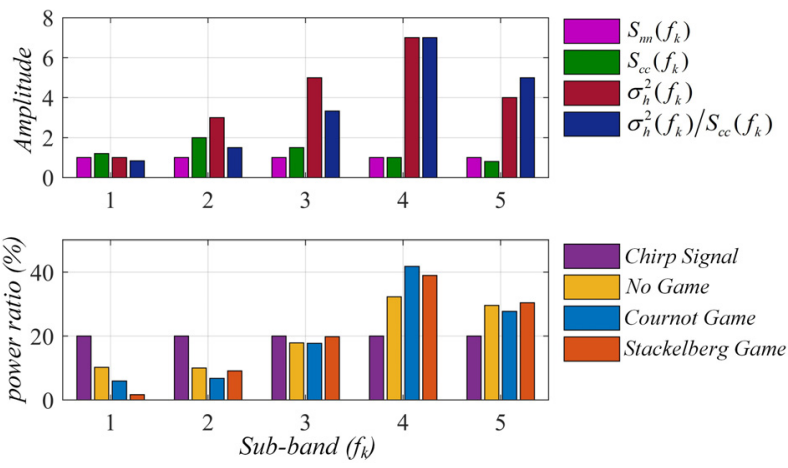

Fig. 5. Radar power allocation for different frequency domain waveforms.

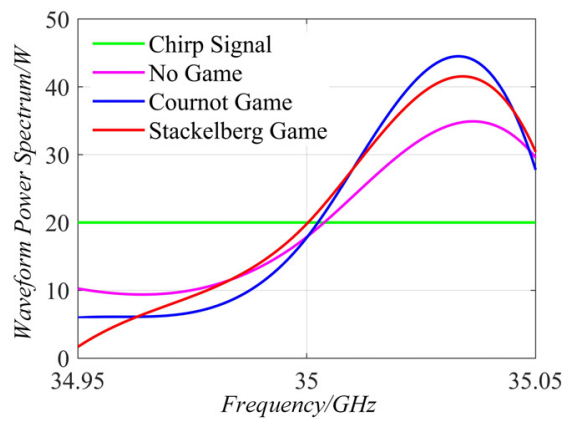

Fig. 6. Radar waveform power spectra of different models.

\begin{tabular}{|c|c|c|}
\hline Model & SINR [dB] & Detection probability \\
\hline Chirp Signal & 8.783 & $36.50 \%$ \\
\hline No Game & 9.287 & $44.84 \%$ \\
\hline Cournot Game & 9.782 & $53.08 \%$ \\
\hline Stackelberg Game & 9.605 & $50.14 \%$ \\
\hline
\end{tabular}

Tab. 2. Radar detection probability in different strategies.

game finally reached equilibrium. It can be seen that the radar will allocate more power to the frequency band with high target-clutter ratio (TCR) to suppress jamming signal, so as to obtain high income. At this point, if jammer changes the strategy, radar will have a better choice, so the game remains equilibrium.

In Stackelberg game, different game sequences result in different information levels. Radar moves first, and jammer optimizes its waveform after observing the radar's strategy. Since the jamming strategy is not known, radar can only predict the jamming waveform and optimize the signal accordingly. Jammer has more posteriori information and a higher game level. It can be seen from Tab. 2 that the output SINR of Stackelberg game is higher than No game and Chirp signal, but lower than Cournot game. Therefore, in radar game model, followers have more battlefield information and late-mover advantage.

\subsection{Jammer Power Variable}

Next, the radar game strategy performance is tested when jamming power changes. Assume that the power jammer varies from $10-30 \mathrm{dBw}$. The radar optimization scheme with jammer's power changes is shown in Fig. 7. With the increase of jamming power in the game process, 

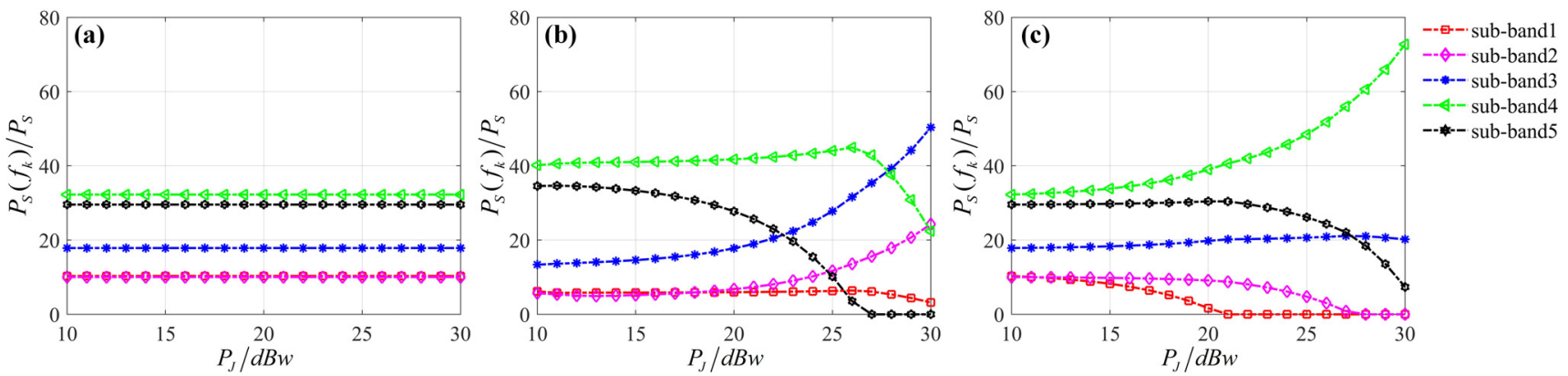

Fig. 7. Radar power allocation strategy. (a) No Game. (b) Cournot Game. (c) Stackelberg Game.

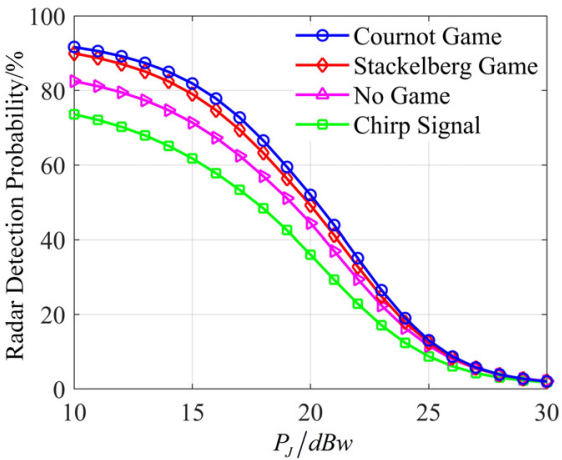

Fig. 8. Radar detection probability curve of different optimized signals.

the high TCR frequency band has been completely covered by jamming, which makes it difficult for radar to obtain target information. In Cournot game, the radar distributes more energy to the band where TCR is low but $\sigma_{\mathrm{h}}{ }^{2}\left(f_{k}\right)$ is high to avoid jamming, such as sub-band 3. In Stackelberg game, radar distributes more energy to high TCR band to counter against jamming, such as sub-band 4 . The radar detection probability curves of different optimized waveforms are shown in Fig. 8.

The most striking result to emerge from Fig. 8 is that compared with No game and Chirp signal, the optimized signal by game theory can achieve higher detection performance. Further analysis shows that the degree of information obtained can affect radar performance in games. In other words, the game has a late-mover advantage in the electronic warfare. It is noteworthy that, for radar, the lower the jamming signal power is, the more obvious the performance improvement by game strategy. At constant transmit power, radar can use game theory to improve the detection performance by optimizing the waveform power spectrum. Unfortunately, if the jamming power is too high, it is difficult for radar to obtain valid results. When the power of jammer is 10 times higher than that of radar $(30 \mathrm{dBw})$, it is difficult to improve the radar performance by optimizing radar waveform. This implies that the optimization of radar performance by game strategy is limited. Overall, radar waveform optimization design based on game theory is significant to improve radar detection performance in electronic warfare. The power difference between radar and jammer will affect the optimization effect.

\section{Conclusion}

Radar waveform power spectrum design is critical to radar system performance. In electronic warfare, the interaction between radar and jammer will affect the radar performance. This paper proposes waveform optimization strategies based on SINR from game theory perspective. Since jammer and radar are completely hostile, their interaction is modeled as two-person zero-sum game. SINR criterion is used in formulating the utility functions. Firstly, the SINR-based radar and jammer unilateral optimization strategy is studied. Secondly, Cournot game and Stackelberg game strategies are designed for different information levels of radar and jammer. In Cournot game, radar and jammer have symmetrical information. We find that Nash equilibrium can be achieved by the iterative water-filling method. In Stackelberg game, the information of radar and jammer is asymmetrical. The two-step water-filling method is used to achieve Stackelberg equilibrium. Finally, the performance of different optimization schemes is compared via simulation. Simulation results reveal that game strategies can bring higher detection probability to radar, and the power difference between radar and jammer will affect the optimization effect. Furthermore, the follower has a latemover advantage in radar and jammer Stackelberg game. This study has shown that without changing transmit power, radar can use game theory to improve the detection performance by optimizing the waveform power spectrum.

\section{Acknowledgments}

This work is supported by the National Natural Science Foundation of China (No. 61302153 and No. 61571456), and the Aeronautical Science Foundation of China (No. 20160196001). All authors thank the reviewers for their valuable comments.

\section{References}

[1] BELL, M. R. Information theory and radar waveform design. IEEE Transactions on Information Theory, 1993, vol. 39, no. 5, p. $1578-1597$. DOI: $10.1109 / 18.259642$ 
[2] PIllai, S. U., OH, H. S., YOULA, D. C., et al. Optimum transmit-receiver design in the presence of signal-dependent interference and channel noise. IEEE Transactions on Information Theory, 2002, vol. 46, no. 5, p. 577-584. DOI: 10.1109/18.825822

[3] KAY, S. Optimal signal design for detection of Gaussian point targets in stationary Gaussian clutter/reverberation. IEEE Journal of Selected Topics in Signal Processing, 2007, vol. 1, no. 1, p. 31-41. DOI: $10.1109 /$ jstsp.2007.897046

[4] ROMERO, R. A., BAE, J., GOODMAN, N. A. Theory and application of SNR and mutual information matched illumination waveforms. IEEE Transactions on Aerospace and Electronic Systems, 2011, vol. 47, no. 2, p. 912-927. DOI: 10.1109/TAES.2011.5751234

[5] AUBRY, A., DEMAI, A., FARINA, A., et al. Knowledge-aided (potentially cognitive) transmit signal and receive filter design in signal-dependent clutter. IEEE Transactions on Aerospace and Electronic Systems, 2013, vol. 49, no. 1, p. 93-117. DOI: 10.1109/TAES.2013.6404093

[6] AHMED, S., ALOUINI, M. S. MIMO-radar waveform covariance matrix for high SINR and low side-lobe levels. IEEE Transactions on Signal Processing, 2014, vol. 62, no. 8, p. 2056-2065. DOI: 10.1109/TSP.2014.2307282

[7] WANG, Y., LI, W., HUANG, G., et al. Time-invariant range-angle dependent beampattern synthesis for FDA radar targets tracking. IEEE Antennas and Wireless Propagation Letters, 2017, vol. 16, no. 1 , p. 2375-2379. DOI: 10.1109/LAWP.2017.2718580

[8] IMANI, S., NAYEBI, M. M., GHORASHI, S. A. Colocated MIMO radar SINR maximization under ISL and PSL constraints. IEEE Signal Processing Letters, 2018, vol. 25, no. 3, p. 422-426. DOI: 10.1109/LSP.2018.2796603

[9] WU, L., BABU, P., PALOMAR, D. P. Transmit waveform/receive filter design for MIMO radar with multiple waveform constraints. IEEE Transactions on Signal Processing, 2018, vol. 66, no. 6, p. 1526-1540. DOI: 10.1109/TSP.2017.2787115

[10] HAYKIN, S. Cognitive radar: A way of the future. IEEE Signal Processing Magazine, 2006, vol. 23, no. 1, p. 30-40. DOI: 10.1109/MSP.2006.1593335

[11] WANG, L., WANG, H., WONG, K. K., et al. Minimax robust jamming techniques based on signal-to-interference-plus-noise ratio and mutual information criteria. IET Communications, 2014, vol. 8 , no. 10 , p. 1859-1867. DOI: 10.1049/iet-com.2013.1054

[12] GOGINENI, S., NEHORAI, A. Game theoretic design for polarimetric MIMO radar target detection. Signal Processing, 2012, vol. 92, no. 5, p. 1281-1289. DOI: 10.1016/j.sigpro.2011.11.024

[13] PANOUI, A., LAMBOTHARAN, S., CHAMBERS, J. A. Game theoretic distributed waveform design for multistatic radar networks. IEEE Transactions on Aerospace and Electronic Systems, 2016, vol. 52, no. 4, p. 1855-1865. DOI: 10.1109/TAES.2016.150378

[14] DELIGIANNIS, A., LAMBOTHARAN, S., CHAMBERS, J. A Game theoretic analysis for MIMO radars with multiple targets. IEEE Transactions on Aerospace and Electronic Systems, 2016, vol. 52, no. 6, p. 2760-2774. DOI: 10.1109/TAES.2016.150699

[15] DELIGIANNIS, A., PANOUI, A., LAMBOTHARAN, S., et al. Game-theoretic power allocation and the Nash equilibrium analysis for a multistatic MIMO radar network. IEEE Transactions on Signal Processing, 2017, vol. 65, no. 24, p. 6397-6408. DOI: 10.1109/TSP.2017.2755591

[16] HAN, K., NEHORAI, A. Jointly optimal design for MIMO radar frequency-hopping waveforms using game theory. IEEE
Transactions on Aerospace and Electronic Systems, 2016, vol. 52, no. 2 , p. 809-820. DOI: 10.1109/TAES.2015.140408

[17] SHI, C., SALOUS, S., WANG, F. et al. Power allocation for target detection in radar networks based on low probability of intercept: A cooperative game theoretical strategy. Radio Science, 2017, vol. 52, no. 8, p. 1030-1045. DOI: 10.1002/2017RS006332

[18] SHI, C., WANG, F., SELLATHURAI, M., et al. Non-cooperative game theoretic power allocation strategy for distributed multipleradar architecture in a spectrum sharing environment. IEEE Access, 2018, vol. 6, no. 1, p. 17787-17800. DOI: 10.1109/ACCESS.2018.2817625

[19] SONG, X., WILlETT, P., ZHOU, S., et al. The MIMO radar and jammer games. IEEE Transactions on Signal Processing, 2012, vol. 60, no. 2, p. 687-699. DOI: 10.1109/TSP.2011.2169251

[20] GAO, H., WANG, J., JIANG, C., et al. Equilibrium between a statistical MIMO radar and a jammer. In IEEE Radar Conference. Johannesburg (RSA), 2015. p. 461-466. DOI: 10.1109/RADAR.2015.7131043

[21] LAN, X., LI, W., WANG, X., et al. MIMO radar and target Stackelberg game in the presence of clutter. IEEE Sensors Journal, 2015, vol. 15 , no. 12 , p. 6912-6920. DOI: 10.1109/JSEN.2015.2466812

[22] BASAR, T., OLSDER, G. J. Dynamic Noncooperative Game Theory. (Vol. 160, Mathematics in Science and Engineering). New York-London (USA): Academic Press, 1982. ISBN: 0-12-080220-1

[23] CARABAllo, M. A., MÁrmol, A. M., MONROY, L., et al. Cournot competition under uncertainty: Conservative and optimistic equilibria. Review of Economic Design, 2015, vol. 19, no. 2 , p. $145-165$. DOI: $10.1007 / \mathrm{s} 10058-015-0171-\mathrm{z}$

\section{About the Authors ...}

Honglin WANG was born in Weihai, China, in 1995. He received the B.E. degree from the Air Force Engineering University, Xi'an, China, in 2017, where he is currently pursuing the MA.Sc. degree. His research interests include radar signal design and electronic warfare system.

Wei LI (corresponding author) was born in Jining, China, in 1978. He received the Ph.D. degree from the National University of Defense Technology, in 2006. His research interests include radar signal processing, cognitive radar waveform design, and radar stealth technology.

He WANG (corresponding author) was born in Beijing, China, in 1995. He received the B.E. degree from the Air Force Engineering University in 2017. His research interests include metasurfaces and antennas design.

Jianye XU was born in Henan, China, in 1992. He received the B.E. degree in Beihang University, Beijing, China, in 2012. His research interests include deep learning and code design.

Junlong ZHAO was born in AnHui, China, in 1995. He received the B.E. degree in Beihang University, Beijing, China, in 2018. His research interests include deep learning and radar waveform design. 